\title{
A hybrid approach for categorizing images based on complex networks and neural networks
}

\author{
Ali Ebrahimi' ${ }^{1}$ Kamal Mirzaie ${ }^{1}$, Ali Mohamad Latif ${ }^{2}$ \\ ${ }^{1}$ Department of Computer Engineering, Maybod Branch, Islamic Azad University, Maybod, Iran \\ ${ }^{2}$ Department of Computer Engineering, Yazd University, Yazd, Iran
}

\begin{tabular}{l} 
Article Info \\
\hline Article history: \\
Received Mar 13, 2021 \\
Revised May 31, 2021 \\
Accepted Jul 1, 2021 \\
\hline
\end{tabular}

Keywords:

Complex network

Key points

Network similarity

Neural network

Small-world network

\begin{abstract}
There are several methods for categorizing images, the most of which are statistical, geometric, model-based and structural methods. In this paper, a new method for describing images based on complex network models is presented. Each image contains a number of key points that can be identified through standard edge detection algorithms. To understand each image better, we can use these points to create a graph of the image. In order to facilitate the use of graphs, generated graphs are created in the form of a complex network of small-worlds. Complex grid features such as topological and dynamic features can be used to display image-related features. After generating this information, it normalizes them and uses them as suitable features for categorizing images. For this purpose, the generated information is given to the neural network. Based on these features and the use of neural networks, comparisons between new images are performed. The results of the article show that this method has a good performance in identifying similarities and finally categorizing them.
\end{abstract}

This is an open access article under the CC BY-SA license.

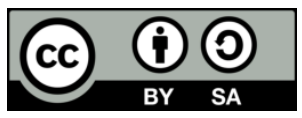

\section{Corresponding Author:}

Kamal Mirzaie

Department of Computer Engineering, Maybod Branch, Islamic Azad University

Maybod, Iran

Email: k.mirzaie@maybodiau.ac.ir

\section{INTRODUCTION}

With the production of the first digital image in 1957 by Russell Kirsch a scientist at the National Research Institute for Standards and Technology (NIST) the world has virtually entered a new era of digital imaging. Rapid development of digital imaging intensified after the advent of microprocessors in the early 1970s. Millions of digital imaging machines have revolutionized conventional photography over the past decade and into the late twentieth century, producing billions of digital images. Due to the huge volume of digital images on Internet and their diversity, large and small digital libraries have been produced around the world, which has led to manage image databases and use tools for image retrieval and classification [1].

The term "content-based image retrieval" dates back to 1992 when Kato used a database based on existing colors and shapes to describe experiments involving automatic image retrieval. Since then, this term has been used to describe the process of retrieving images from a large set based on coordinated visual features. The used techniques, tools, and algorithms apply methods such as statistics, pattern recognition, signal processing, and computer vision. The first commercial content-based image retrieve (CBIR) system developed by immersed boundary method (IBM) was called query by image content (QBIC) [2].

Textual information about images can be easily searched using existing technologies, but it is a human-dependent method to manually describe the information of each image in the database. Therefore, this method is practically not applicable for very large databases or images that are generated automatically. The 
original CBIR system was designed to search databases based on color, texture, and shape properties. After the development of these systems, the need for a user-friendly interface became apparent. Therefore, CBIR includes a plan that tries to meet the needs of the user and perform his searches. Conventional methods for image retrieval include: semantic evaluation, feedback method (two-way communication between machine and human), machine learning, query methods, content comparison using image distance measurement, texture, correlation matrix, and wavelet transform are all used [2].

So far, different methods with advantages and disadvantages have been introduced to identify image patterns. In recent years, the recognition of texture and the classification of images by color and form have been in the center of attention. For example, Ramesh introduced a histogram-based system that uses histogram characteristics to categorize images [3]. Moreover, Shirazi et al. [1] compared the various methods used by the vector machine for categorizing the images. Shakoor et al. [2] used a local entropy method to identify the image features and used them for categorization.

As mentioned, one of the most important features of any image is its key points. In other words, key points are one of the most important indicators of any image, and so far, various algorithms have been designed to identify them, some of which are more important or are used more. Edges can be used as one of the key points in identifying and classifying images. The purpose of identifying edges is to mark points in an image where the intensity of light changes dramatically. Rapid changes in image properties usually represent important events and changes in environmental characteristics [3].

The basic theory in most edge detection methods is the calculation of a local derivative operator. The size of the first derivative of the image at each point is equal to the size of the gradient. The second derivative is also obtained using the Laplace approximation. If we consider an edge as a change in brightness seen over several pixels, edge detection algorithms generally calculate a derivative of this change in brightness. After calculating the derivative, the next step is to apply a threshold to discover the points that are part of an edge. The lower the threshold, the more lines are detected and the more sensitive the results are to noise and unrelated image features. On the other hand, a high threshold may miss weak lines or parts of lines. In most works done to retrieve images or determine the similarity between two images, edge recognition has been used to identify key points. For this purpose, edge finding algorithms such as Harris, Shi, Sift, and Surf. have been used. As the name implies, these algorithms are used to identify edges or corners of an image that are more important than other points. Each of these algorithms has been used in specific cases, but in general, each has been created to complete the shortcomings of other existing algorithms and to create a more accurate algorithm for edge and key point detection, although a complete and accurate edge algorithm for all images has not been designed yet [4].

For example, the scale invariant feature transform (SIFT) algorithm is used in tasks such as matching different faces of a body or scene, and identifying objects. The characteristics obtained are somewhat constant regardless of the image scale, rotation, change of view, and lighting variations [4]. The speed-up robust feature (SURF) algorithm is also a feature and descriptor detection method, and its standard version is much faster than SIFT. The algorithm with an accurate and decisive approximation is used to detect points, which can be calculated with the help of the integral. SURF is used to detect objects, individuals, or faces to rebuild 3D scenes for tracking objects [5]. Another algorithm is the Shi-Tomasi method, in which the minimum of the values is computed. Based on the matrix theory, these values are used to identify the edges and key points [6]. One of the other algorithms is the Harris method, which is introduced by Harris and Stephens and improved the moravec corner detector method [7].

Tomasi and Kanade [6], founded the theory of graphs in 1736 by solving the problem of the Konigsberg Stairs. But James Joseph Sylvester was the first to call these mathematical models graphs in 1878. Graphs play an important role in simplifying images and their understanding by computer systems. As we know, each graph consists of a number of nodes that are connected to each other by edges. Graphs have different models (directed graph, undirected graph, mixed graph, weighted graph, oriented graph, and cycle graph).

Graph similarity determination is particularly challenging as graphs fail to be naturally represented or converted into vector spaces. Likewise, graphs intended for pattern recognition have many edges and nodes, rendering them are busy. Graph similarity can be measured in a variety of ways. Main methods are based on graph spacing in graph edit distance (GED) [8], [9], the graph core [10]-[12], and graph matching and topological description methods [12]. Sanfeliu and Fu [8] pioneered the notion of GED, which is being widely used. Graphs can be typically converted to string sequences, with their direction utilized to compare these strings [13]. Using the Levenshtein distance, Hancock calculated string similarity [14]. Tang converted the graphs to the string, and based on the theory of the catch graph, and using the dynamic time deviation of dynamic time warping (DTW), calculated the similarity of the fibers [15]. This problem was formulated by Kelly and Hancock [13] to increase the likelihood of series-mediated binary graph alignment. The Gao algorithm distance (GAD) provided a histogram based on GED calculation for two graphs [16]. 
Graph similarity can also be measured by calculating the similarity between a couples of frequentlyused pattern-based graphs. There are simple as well as complex patterns. Core graph function development practices have been thoroughly examined in the best possible way. These methods can be broadly categorized based on sub-classes [8], shortest paths [5], random steps [15] and sub-graphs [12].

In stochastic nuclei, graph similarity is measured by counting basic steps between each pair. This kind of core is usually very complex and shaky. By replacing the steps with paths, Bargwardt et al. introduced the shortest nucleus of the path on the graphs [11]. The main goal is to map the graphs into vector spaces in which important dimensions are concerned. Furthermore, various image processing methods can be investigated by reviewing the manifold method if a given graph can be embedded in manifold template space. Nevertheless, a number of counterparts are applicable in creating low-dimensional spaces for a graph set. This can be accomplished by representing graphs using pattern vectors. This problem can be solved using topological descriptors [16], [17]. These methods first map out each graph and then use the spacing and metric on the vector to study the graphs. Many actions have been taken to illustrate the topology of the graph. For example, research [18], [19] mapped the structure of a graph into a fixed-length design through the spectral graph theory. They exploited vectors derived from graphs to manage the calculation of the distance between two graphs and evaluate their structures according to principal component analysis (PCA) and multidimensional scaling (MDS) techniques.

The Gao graph with the histogram schematics [20] is similar to the Luo method [18]. In this method, the cost function or node similarity level in similar vectors in the two graphs cannot be formulated. The determination of both cases may be tricky, rendering the method disadvantageous mainly because it fails to examine graph structures in accordance with histogram plotters. The present article used the method of displaying complex networks. Complex networks are characterized as taking advantage of the combination of graph theory, probability theory. Recent efforts have led to the use of complex networks in various applications and fields. For example, Khani and Mirzaei used the structure of the small network for simulating cancer as a multi-functional approach to biological systems [20].

Liu et al. [21] provided a time series clustering given the high dimensions of multivariate time series and most previous work focusing on univariate time series clustering, a new method based on principal component analysis to achieve clustering. Multivariate time series are presented with more speed and accuracy. They are inspired by the traditional k-Means clustering method and have used the detailed algorithm of the proposed Mc2PCA method to analyze and cluster multivariate time series.

Lü et al. [22] provided a new technology to research the problem of the synchronization transmission of spiral wave and turbulence between uncertain time-delay neuronal networks with different node numbers and topologies. Recently novel ideas about advancing evolutionary dynamics towards new phenomena including many new topics, even the dynamics of equivalent social networks have been proposed. In fact, they include more advanced complex networks and incorporate them with the coupled map lattices (CMLs), which are usually used for spatiotemporal complex systems simulation and analysis [23], [24].

This study graphically represented the structure of a complex network-based grid. The graph structure is initially mapped as a complex network, followed by the description of the graph structure by deriving the topological and dynamic complexity of the complex network. These features were extracted using neural networks and will be used to categorize images.

The study of complex networks is a young and active area of scientific research (since 2000) inspired largely by empirical findings of real-world networks such as computer networks, biological networks, technological networks, brain networks, climate networks and social networks. Euler's solution of the Seven Bridges of Konigsberg problem is considered to be the first true proof in the theory of networks.

Complex networks can be categorized into three models: i) random networks, ii) small-world networks, and iii) scale-free networks. In the random model, which is the simplest model, the edges are added randomly. A small-world network is a type of mathematical graph in which most nodes are not neighbors of one another, but the neighbors of any given node are likely to be neighbors of each other and most nodes can be reached from every other node by a small number of hops or steps. Specifically, a smallworld network is defined to be a network where the typical distance L between two randomly chosen nodes (the number of steps required) grows proportionally to the logarithm of the number of nodes $\mathrm{N}$ in the network.

A scale-free network is a type of random network in which some nodes have been removed. In this network, a small number of nodes are very important and by removing them, the network is broken. These nodes are called poles. Social networks are scale-free networks.

The complex network edges as a graph can be binary, weight-oriented, and directional. In this article, only an undirected model was checked. The non-directional complex network model was usually represented by the $\mathrm{W}$ matrix, where $\mathrm{w}(\mathrm{i}, \mathrm{j})$ is the weights that connect node $\mathrm{i}$ to node $\mathrm{j}$. 
The topology and complexity of the network can be detected using the measurements taken from the graph theory and also for complex network research. Every complicated network has a specific topological design that determines how to connect. Accordingly, complex networks can be transformed depending on what measures are used, facilitating the derivation and classification of suitable topological designs .

Grade distribution is an important attribute of the vertex in the graph. Given vertex degrees, many measures can be formulated, with the two most straightforward measurement techniques as in (1) and (2):

$$
\begin{aligned}
& K_{\max }=\max _{i} K_{i} \\
& K_{\text {avg }}=\frac{1}{n} \sum_{i=1}^{n} K_{i}
\end{aligned}
$$

where $\mathrm{K}_{\mathrm{i}}$ indicates the degree of node $\mathrm{i}$. Structural and dynamic networks can be principally characterized by connectivity degree distribution, including: i) entropy, ii) energy, and iii) average connection degree.

The entropy of connectivity degree distribution can be determined as shown in (3):

$$
K_{j d}\left(k, k^{\prime}\right)=-\sum_{k, k^{\prime}=1}^{K_{\max }} p\left(k, k^{\prime}\right) \log \left(p\left(k, k^{\prime}\right)\right)
$$

The normal distribution-associated energy can be determined as shown in (4):

$$
E=\sum_{k, k^{\prime}=1}^{K_{\max }} p\left(k, k^{\prime}\right)
$$

One way to detect the loop is through the clustering coefficient, which is beneficial for network analysis like degree. Two different clustering coefficients are typically used, the first of which, according to the definition of nondirectional networks, is as in (5):

$$
E=\frac{3 N_{\Delta}}{N_{3}}
$$

where $\mathrm{N}_{\Delta}$ represents the number of triangles on the grid and $\mathrm{N}_{3}$ represents the triangles.

A triangle has three vertices, with an edge between each pair, a triangular connected set of three vertices, whose two vertices are adjacent to a third one (i.e., central vertex). Clustering factors can be obtained for a grid by calculating the mean clustering factor for each vertex. The clustering factor of vertex $i$ can be measured as in (6):

$$
C_{i}=\frac{N_{\Delta}(i)}{N_{3}(i)}
$$

where $\mathrm{N}_{\Delta}(\mathrm{i})$ denotes the number of triangles with vertices $\mathrm{i}$ and $\mathrm{N}_{3}(\mathrm{i})$ denotes the number of triangles, i.e., the central vertex. The clustering coefficient of a network can be defined as in (7):

$$
\bar{C}=\frac{1}{N} \sum_{i} C_{i}
$$

The distance is an important size, depending on the overall network structure. We can measure the network as the mean of the shortest distance by calculating the mean distance of the shortest line (path) for each pair of vertices, such as (8):

$$
d_{G}=\frac{1}{N(N-1)} \sum_{i+j} d_{i j}
$$

$d_{i j}$ represents the shortest line distance from vertex $i$ and vertex $j$. 


\section{DISPLAY OF COMPLEX NETWORK PROPERTIES}

This section initially models a graph by a complex network and, consequently, extracts the dynamic and topological properties of the above network to detect the graph structure, which is named "Display of complex network properties of a complex network properties extraction (CNCRG) graph". Thus, the distance between CNCRGs can be taken as a similarity index between the two graphs. Regarding the relation between graph edges constituting the basic elements of the graph structure, CNCRGs would help identify the differences between the two graphs. This can be associated with GED, which takes into account differences in the binding of the graphs through the order of editing operations, including adding, deleting, and replacing the edges. The CNCRG is completely independent of the cost function.

\section{PROPOSED APPROACH BASED ON COMPLEX NETWORKS AND NEURAL NETWORKS}

In this paper, the proposed method was a combination of complex networks and neural networks. Using the complex network, the properties were extracted and then, the neural network was used to classify. Assume graph $\mathrm{G}=(\mathrm{V}, \mathrm{E})$. Here, $\mathrm{E}$ and $\mathrm{V}$ indicate the set of edges and vertices, respectively. $|\mathrm{E}|$ and $\mathrm{V} \mid$ denote the number of edges and nodes of the graph $\mathrm{G}$, respectively. The network model can be defined as $\mathrm{GN}=\left(\mathrm{V}_{\mathrm{N}}, \mathrm{E}_{\mathrm{N}}\right)$ for the $\mathrm{G}$ graph. Each edge of the graph $\mathrm{G}$ is a vertex $\mathrm{GN}$. The weighting vector for each vertex can be measured by evaluating the relationship between and geometric position of the edges, as shown in Figure 1, the weighting vector for vertex i can be measured as seen in (9):

$$
e_{i}=\left(l_{i}, d_{i}, d_{1 i}, d_{2 i}, x_{i}, y_{i}\right)
$$

where $l_{i}$ represents edge length, $d_{i}$ represents the distance between the edge center and graph center (Point o), $\mathrm{d}_{2 \mathrm{i}}$ and $\mathrm{d}_{1 \mathrm{i}}$ the intervals from the beginning of the edge to the center of the graph (point $\mathrm{o}$ ) and from edge end to graph center (point $\mathrm{o}$ ) and $\mathrm{x}_{\mathrm{i}}$ and $\mathrm{y}_{\mathrm{i}}$ are the center point coordination of the edges.

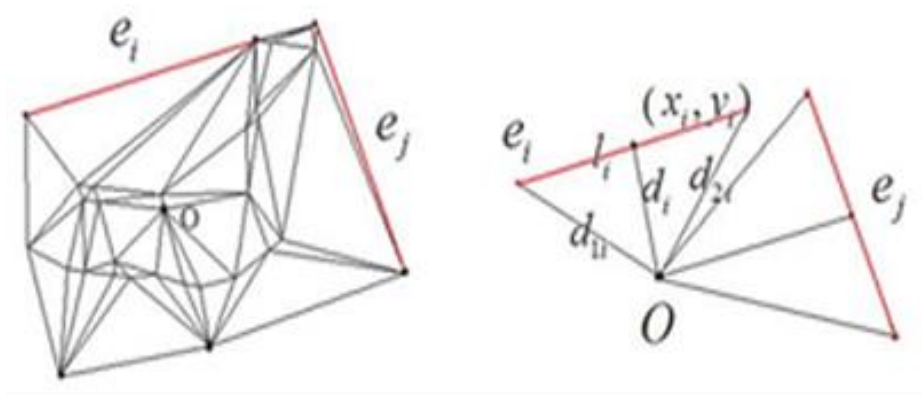

Figure 1. Schematic representation of a graph based on a complex network

The network is formed by a set of nondirectional edges $E$ that connects each pair of vertices. The value of the edge of vertex $i$ to vertex $j$ can be calculated using the Euclidean distance between $e_{i}$ and $e_{j}$ :

$$
W_{i j}=d\left(e_{i}, e_{j}\right)
$$

Therefore, the network can be mapped as $|\mathrm{E}| \times|\mathrm{E}|$ and show the weight $\mathrm{w}$ with the matrix.

$$
W_{i j}=W\left(\left[e_{i}, e_{j}\right]\right)
$$

And then normalize it to form:

$$
W=\frac{W}{\max \left(w_{i j} \in W\right)}
$$

The network model $G N=\left(V_{N}, E_{N}\right)$ is initially a regular network that connects the set of edges of $E_{N}$ to each node of the network; although however, this regular network does not offer any good useful features for the program. Hence, this regular network must be transformed into a complex network with appropriate characteristics. Here we carry this transition using the method applied by Tang et al. [15] and define the 
threshold t. This transition is performed to create a new edge set for the network by choosing a subset of performed the $\mathrm{E}_{\mathrm{N}}$ of $\mathrm{E}_{\mathrm{N}}$ which eliminates the need for the edges of the $\mathrm{E}_{\mathrm{N}}$ to have a lower weight than the threshold of t. This can be defined as:

$$
G_{C N}^{t}=\delta\left(G_{N}, t\right)=\left\{\begin{array}{l}
w_{i j}=0 \text { if } w_{i j} \geq t \\
w_{i j}=1 \text { if } w_{i j}<t
\end{array}\right.
$$

where $t$ varies between $t_{0}$ and $t_{f}$ and the first and final thresholds $\left(t_{f}, t_{0}\right)$ are user-defined.

Given a graph $G=(V, E)$, we first model the graph structure using network $G_{N}$ and then extract measurements from networks $\mathrm{G}_{\mathrm{CN}}^{\mathrm{t}}=\delta\left(\mathrm{G}_{\mathrm{N}}, t\right)$ by varying the threshold $\mathrm{t}$. Afterwards, our CNCRG is performed as the concatenation of these measurements. Three distinct feature vectors are proposed, i.e., degree descriptors, joint degree descriptors, and clustering-distance descriptors.

Using formulas described in (1)-(3), $\varphi_{\mathrm{t}}$ denote degree descriptors as follows:

$$
\varphi_{t}=\left(K_{\max }(t), K_{a v g}(t), K_{d}(t)\right)
$$

Using formulas described in (3) and (4), $\gamma_{t}$ denote joint degree descriptors as follows:

$$
\gamma_{t}=\left(H_{j d}(t), E_{j d}(t)\right)
$$

Using formulas described in (7) and (8), $\xi_{\mathrm{t}}$ denote clustering-distance as follows:

$$
\xi_{t}=\left(\bar{C}(t), G_{d}(t)\right)
$$

The final feature vector for $\mathrm{G}_{\mathrm{CN}}^{\mathrm{t}}$ can be computed as:

$$
f_{\mathrm{t}}=\left[\varphi_{\mathrm{t}}, \gamma_{t}, \xi_{t}\right.
$$

with the feature vector for $\mathrm{G}_{\mathrm{CN}}^{\mathrm{t}}$, our CNCRG can be computed as the concatenation of $f_{\mathrm{t}}$ at different stages of the evolution of the network, according to the following:

$$
C N C R G=f_{t 0}, f_{t 1}, \ldots, f_{t j}
$$

This approach is used to convert the regular network to the complex network, as illustrated in Figure 2.
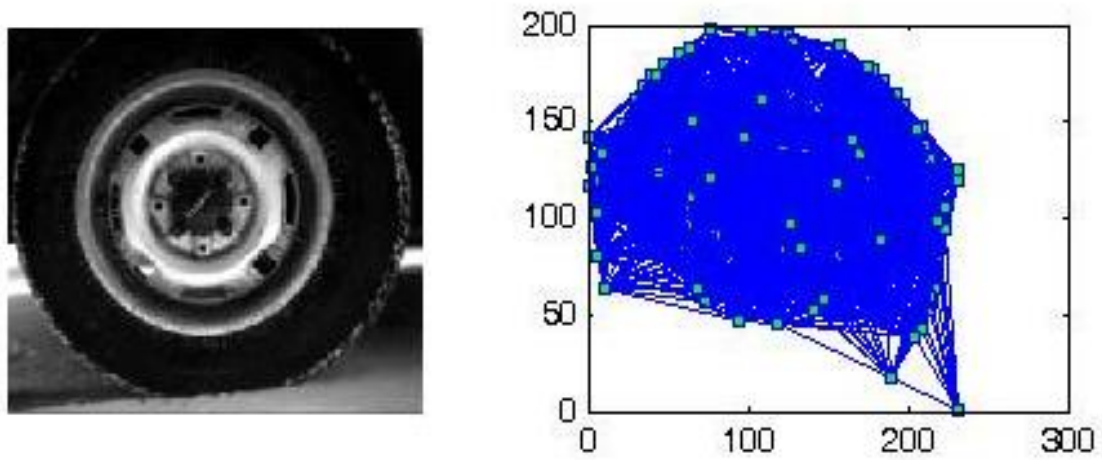

Figure 2. The image of a shape and its complex network

In [14], they showed the complex network $G_{c_{n}}^{t_{0}}$ is as suitable for the small-world model. This complex network model has two main characteristics: i) high clustering coefficient and ii) small-world property that can be based on the discussed issues. The clustering coefficients listed in (7) can be used to evaluate the characteristics of high clustering coefficients. The small-world property is quantified based on whether an average shortest distance is present on the network (8). 
In this paper, the four algorithms introduced in the previous section for identifying the edge were used to obtain the best pattern for identifying the corners and key points, and its output was examined for two standard images (camera man and house) in MATLAB software. The results of the implementation of the algorithms and their comparison together indicated that the Harris algorithm has higher accuracy and speed, and thus, it is used in the rest of the stages. It shows in Table 1.

Table 1. The comparison of the number of nodes and edges with two standard images

\begin{tabular}{cccccc}
\hline \multicolumn{2}{c}{ Number of Edges } & \multicolumn{2}{c}{ Number of Nodes } & Method & Seq. \\
House & Cameraman & House & Cameraman & & \\
\hline 5050 & 16836 & 101 & 184 & Harris & 1 \\
19900 & 27150 & 200 & 258 & Shi & 2 \\
63903 & 390286 & 358 & 883 & SIFT & 3 \\
4186 & 16110 & 92 & 179 & SURF & 4 \\
\hline
\end{tabular}

There are several ways to identify neighbors in order to network and connect with them. For example, Leandro et al. [25] use the tourist walk method. In this method, first each node is randomly selected and then the nearest node is selected based on the Euclidean distance between them. Then the edge between these two nodes is drawn. Now for the new node, the nearest node other than the previous node is selected. This method continues until they reach one of the pre-selected points. Eventually all of these networks will be merged and a new network will be created. Tang et al. [19] used the k-means algorithm to identify neighboring nodes.

This paper used the k-nearest neighbors (K-NN) algorithm to identify neighbors and uses triangles to construct triangles; in other words, after identifying the nodes adjacent to one node, the edge was formed between the main noun and the neighbors. Finally, after performing this process for all nodes, the parameters related to the shortest path, clustering coefficient, and other required parameters were extracted. Figure 3 shows the steps of the proposed approach.

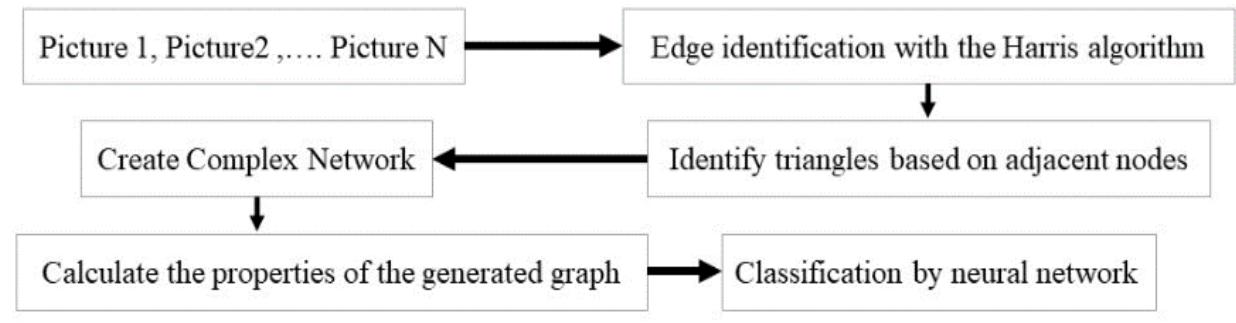

Figure 3. Classification of images with the proposed hybrid approach

- Step 1-Edge detection and extraction of key points of the image

- Step 2-Segmentation of points using Euclidean distance to form triangular areas

- Step 3-After identifying nodes and their relationship, the network is formed using specified nodes and the relationships between them.

- Step 4-complex network properties extraction (CNCRG) for image description

- Step 5-The information obtained from the previous step is given for neural network training. For this purpose, $70 \%$ of information is used for network training and $15 \%$ for verification and the remaining $15 \%$ for testing.

\section{RESULTS}

\subsection{Performance evaluation of the proposed method}

System testing is conducted on various sample images available in MATLAB software, for example, the corresponding results for two images with their outputs to form a complex network are shown in Figure 4 and Figure 5. Assume that our image is a matrix $m * n$, where $m$ and $n$ indicate the number of rows and columns, respectively. For assigning a number to pixel A, which is located in row i and column $\mathrm{j}$, where $1<\mathrm{i}<\mathrm{m}$ and $1<\mathrm{j}<\mathrm{n}$, the number is obtained by the following relation to the pixel: 


$$
k=(i-1) * n+j
$$

For better comparison, the edges of the image are identified according to Harris and SURF algorithms, which part of the results is shown in Tables 2 and 3.
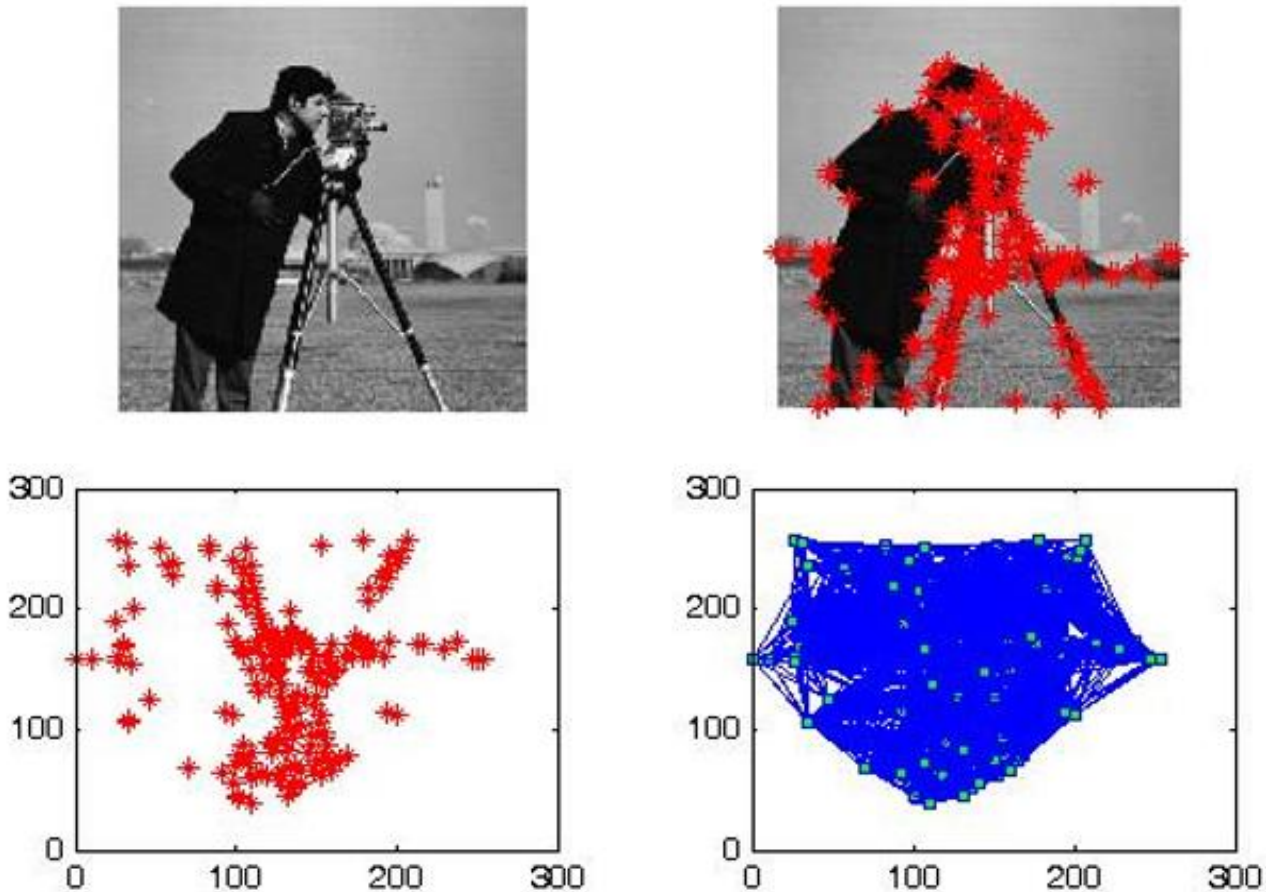

Figure 4. Result of cameraman image processing
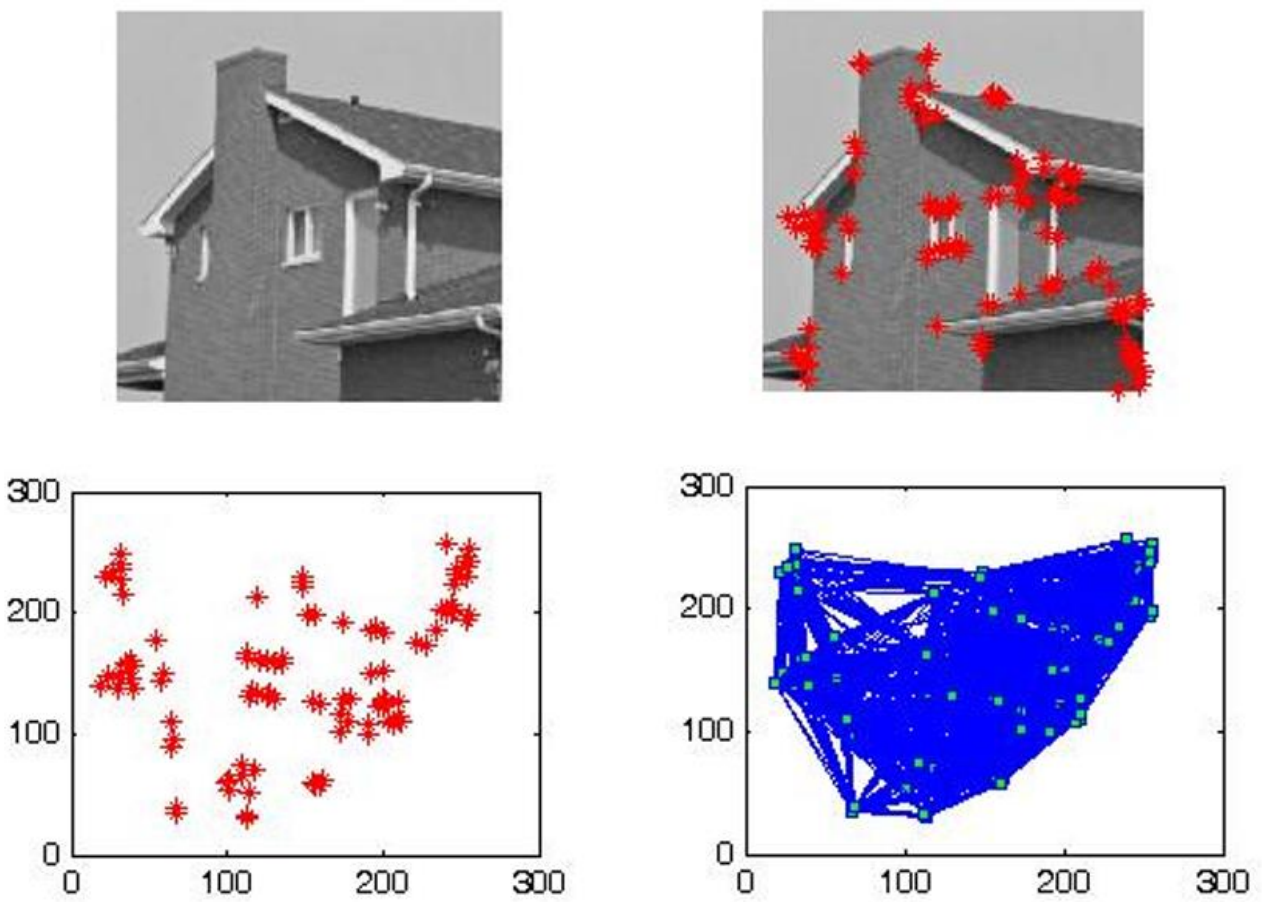

Figure 5. Result of home image processing 
Table 2. Part of sample information with Harris algorithm

\begin{tabular}{cccccc}
\hline Picture & Node No. & Triangle No. & Clustering & Distance Avg. & Weight \\
\hline 1 & 40 & 15 & 3.9 & 59.86 & 96 \\
2 & 38 & 15 & 3.7 & 58.2 & 87 \\
3 & 43 & 16 & 3.97 & 60.07 & 97 \\
4 & 41 & 15 & 3.83 & 59.98 & 89 \\
\hline
\end{tabular}

Table 3. Sample part information with SURF algorithm

\begin{tabular}{cccccc}
\hline Picture & Node No. & Triangle No. & Clustering & Distance Avg. & Weight \\
\hline 1 & 69 & 22 & 3.9 & 72.71 & 97 \\
2 & 62 & 20 & 3.7 & 70.32 & 93 \\
3 & 78 & 27 & 3.97 & 75.43 & 98 \\
4 & 65 & 23 & 3.83 & 71.3 & 94 \\
\hline
\end{tabular}

Then information of $\mathrm{x}$ and $\mathrm{y}$ pixels and their numbers were stored in a table. Then, Euclidean distance between them was calculated, and the output was stored in a matrix containing the number of pixels, and the distance between them. Then, based on the closest neighboring algorithm, the nodes were identified, and practically triangular triangles were formed. Then, the network properties were obtained from the formation of graphs based on triangles.

The above information normalized and output data for each image, including the number of identified nodes and triangles, the clustering coefficient, the average shortest distance, and finally, the normalized weight were stored in a table. This information was then transferred to the neural network for classification. For the initial test, the synthetic sample was first used for testing. An artificial dataset is a set of images of a home model using a set of images. Adjacent houses have been achieved with a 2-degree rotation. The total number of images was 180 images, with the grid diagram shown in Figure 6 . This suggests a similar node number but different edge structures of the graphs .
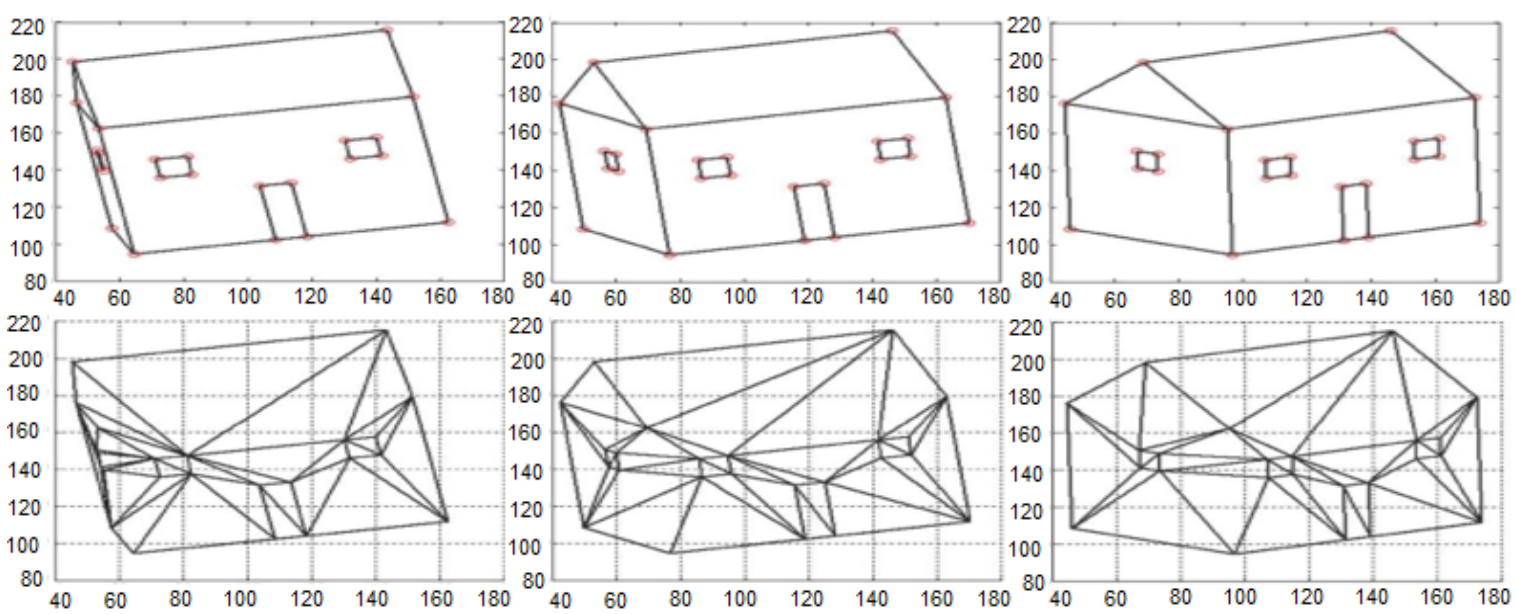

Figure 6. Sequence of the model house with feature points in the top row and the associated Delaunay diagram with the Harris algorithm

As for other complex network parameters, the situation was the same, and the case was approximately equal to the original image values. The reason for the difference was the rotation of the images. To test the system, the coil image set consisted of two classes, and each class consisted of 72 images, including duck and box images. The images of these two sets had a 5-degree rotation angle around the Y-axis. As previously stated, the table for each image was provided and stored in a table for neural network training.

The results of the duck image with the dual algorithm discussed in Figures 7 and 8 are shown for the box image in Figures 9 and 10. The results of the work on these images illustrated the better performance of the Harris algorithm. Besides, the similarity for the box-related class was even better due to the box shape structure and the clearness of the corners in each rotation, while the information for the duck image was distinct, and the number of edges varied in each segment. 

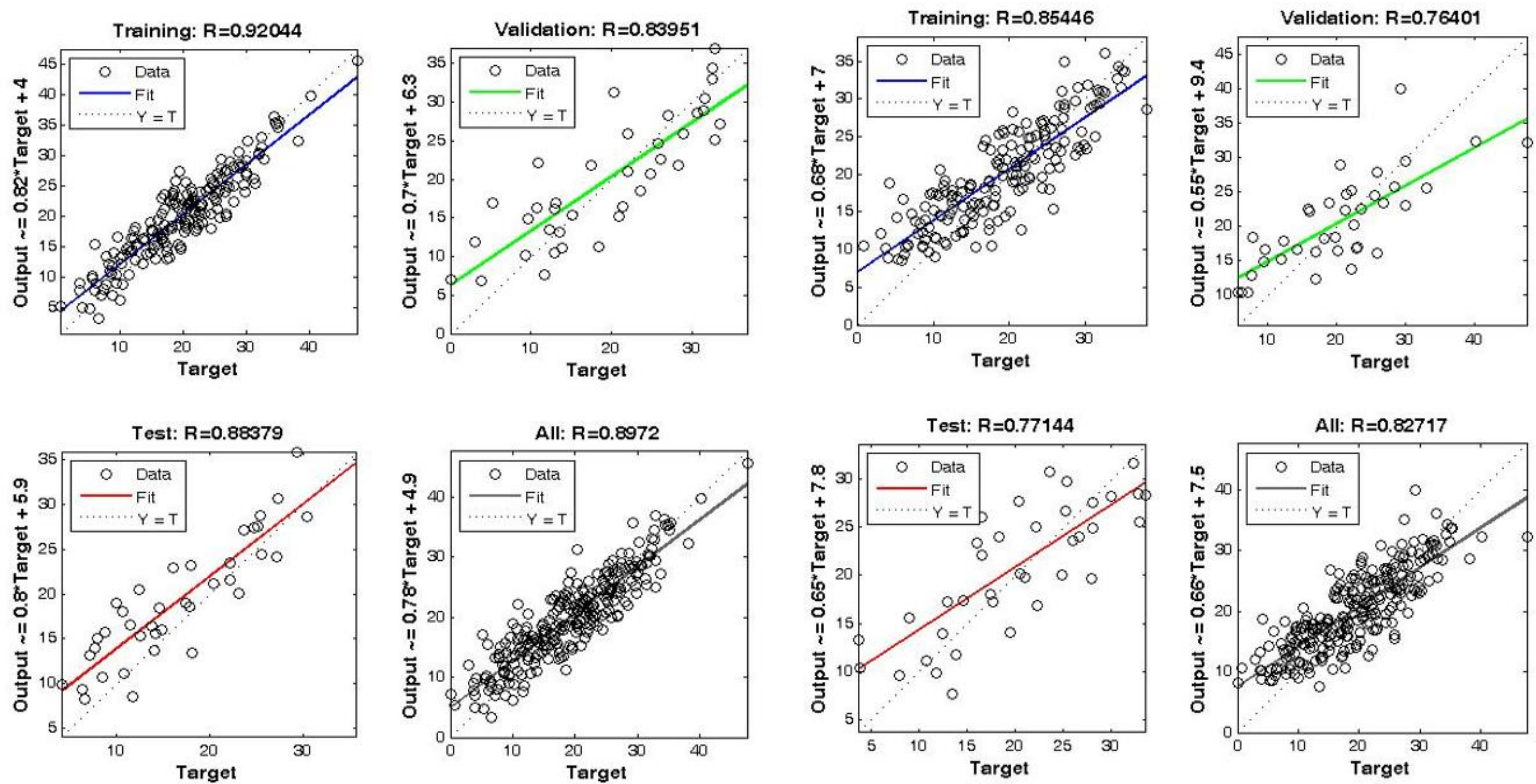

Figure 7. Results for duck image with harris algorithm

Figure 8. Results for duck image with SURF algorithm
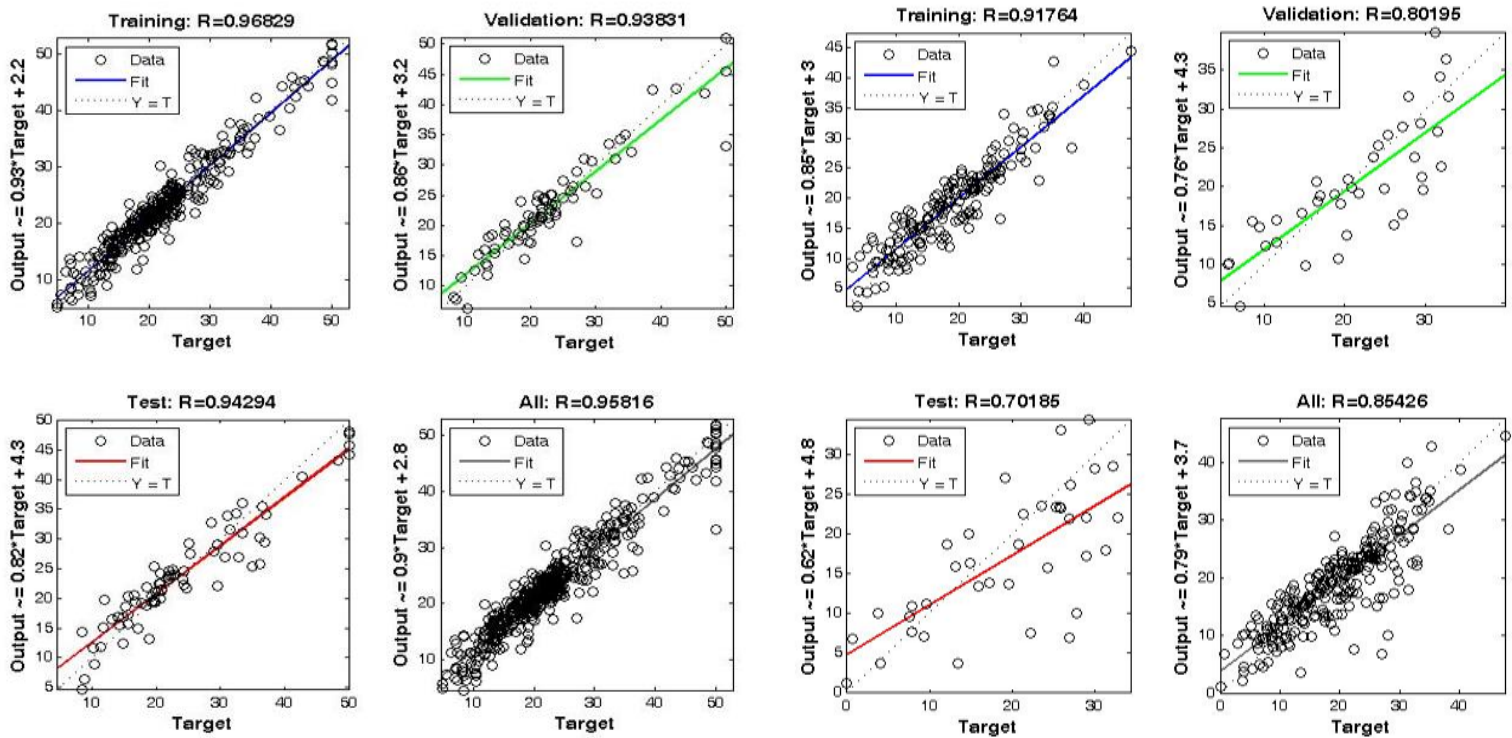

Figure 9. Results for box image with Harris algorithm

Figure 10. Results for box image with SURF algorithm

\section{CONCLUSION}

The present study introduced a hybrid method based on complex and neural networks for image classification. For this purpose, the key points of the images were first identified. By identifying key points using different images of an object at different angles and using the edge-matching algorithm, it identified the edges and the formation of triangles. The image is represented as a graph, and by converting the graph into a complex grid, the image looks like a complex grid. By converting the image into a complex grid, the parameters associated with it can be calculated. Finally, the calculated information was fed to the neural network, and it was determined that these parameters are suitable properties for comparing and classifying images. It is recommended to use more appropriate approaches and methods for classification by improving the neural network learning process or similar methods. 


\section{REFERENCES}

[1] S. H. Shirazi, N. U. A. Khan, A. I. Umar, M. I. Razzak, S. Naz, and B. AlHaqbani, "Content-based image retrieval using texture color shape and region," International Journal of Advanced Computer Science and Applications, vol. 7, no. 1, 2016, doi: 10.14569/IJACSA.2016.070156.

[2] M. H. Shakoor and F. Tajeripour, "Local entropy pattern for feature extraction of texture images," Machine Vision and Image Processing, vol. 3, no. 2, pp. 73-85, 2017.

[3] R. K. Lingadalli and N. Ramesh, "Content based image retrieval using color, shape and texture," International Advanced Research Journal in Science, Engineering and Technology, vol. 2, no. 6, pp. 40-45, 2015, doi: 10.17148/IARJSET.2015.2610.

[4] D. G. Lowe, "Object recognition from local scale-invariant features," in Proceedings of the Seventh IEEE International Conference on Computer Vision, 1999, vol. 2, pp. 1150-1157, doi: 10.1109/ICCV.1999.790410.

[5] H. Bay, A. Ess, T. Tuytelaars, and L. Van Gool, "Speeded-up robust features (SURF)," Computer Vision and Image Understanding, vol. 110, no. 3, pp. 346-359, Jun. 2008, doi: 10.1016/j.cviu.2007.09.014.

[6] C. Tomasi and T. Kanade, "Detection and tracking of point features," Pattern Recognition, vol. 37, pp. 165-168, 2004.

[7] C. Harris and M. Stephens, "A combined corner and edge detector," in Procedings of the Alvey Vision Conference 1988, 1988, pp. 147-151, doi: 10.5244/C.2.23

[8] A. Sanfeliu and K.-S. Fu, “A distance measure between attributed relational graphs for pattern recognition," IEEE Transactions on Systems, Man, and Cybernetics, vol. 13, no. 3, pp. 353-362, May 1983, doi: 10.1109/TSMC.1983.6313167.

[9] A. Torsello and E. R. Hancock, "Computing approximate tree edit distance using relaxation labeling," Pattern Recognition Letters, vol. 24, no. 8, pp. 1089-1097, May 2003, doi: 10.1016/S0167-8655(02)00255-6.

[10] K. M. Borgwardt and H. Kriegel, "Shortest-path kernels on graphs," in Fifth IEEE International Conference on Data Mining (ICDM'05), 2005, pp. 74-81, doi: 10.1109/ICDM.2005.132.

[11] J. Ramon and T. Gartner, "Expressivity versus eciency of graph kernels," in Proceedings of the First International Workshop on Mining Graphs, Trees and Sequences, 2003, pp. 65-74.

[12] N. Shervashidze, S. V. N. Vishwanathan, T. H. Petri, K. Mehlhorn, and K. M. Borgwardt, "Efficient graphlet kernels for large graphs comparison," in 12th International Conference on Artifical Intelligence and Statistics (AISTATS), 2009, pp. 488-495.

[13] A. Robles-Kelly and E. R. Hancock, "Graph edit distance from spectral seriation," IEEE Transactions on Pattern Analysis and Machine Intelligence, vol. 27, no. 3, pp. 365-378, Mar. 2005, doi: 10.1109/TPAMI.2005.56.

[14] R. Myers, R. C. Wison, and E. R. Hancock, "Bayesian graph edit distance," IEEE Transactions on Pattern Analysis and Machine Intelligence, vol. 22, no. 6, pp. 628-635, Jun. 2000, doi: 10.1109/34.862201.

[15] J. Tang, C. Zhang, and B. Luo, "A new approach to graph seriation," in First International Conference on Innovative Computing, Information and Control, 2006, vol. 3, pp. 625-628, doi: 10.1109/ICICIC.2006.385

[16] X. Gao, B. Xiao, D. Tao, and X. Li, "Image categorization: graph edit direction histogram," Pattern Recognition, vol. 41, no. 10, pp. 3179-3191, Oct. 2008, doi: 10.1016/j.patcog.2008.03.025.

[17] H. Zhou, J. Li, J. Li, F. Zhang, and Y. Cui, "A graph clustering method for community detection in complex networks," Physica A: Statistical Mechanics and its Applications, vol. 469, pp. 551-562, Mar. 2017, doi: 10.1016/j.physa.2016.11.015.

[18] B. Luo, R. C. Wilson, and E. R. Hancock, "Spectral embedding of graphs," Pattern Recognition, vol. 36, no. 10, pp. 2213-2230, Oct. 2003, doi: 10.1016/S0031-3203(03)00084-0.

[19] J. Tang, B. Jiang, C.-C. Chang, and B. Luo, "Graph structure analysis based on complex network," Digital Signal Processing, vol. 22, no. 5, pp. 713-725, Sep. 2012, doi: 10.1016/j.dsp.2012.04.011.

[20] H. Khani and K. Mirzaei, "Modeling and simulation of cancer as a biological system with multi-agent systems approach based on complex network theory," in Iranian Intelligent Systems Conference, 2013, pp. 444-449.

[21] H. Li and Z. Liu, "Multivariate time series clustering based on complex network," Pattern Recognition, vol. 115, Jul. 2021, doi: 10.1016/j.patcog.2021.107919.

[22] L. Lü, L. Ge, L. Gao, C. Han, and C. Li, "Synchronization transmission of spiral wave and turbulence in uncertain time-delay neuronal networks," Physica A: Statistical Mechanics and its Applications, vol. 525, pp. 64-71, Jul. 2019, doi: 10.1016/j.physa.2019.03.054.

[23] A. Menc, D. Pająk, and P. Uznański, "Time and space optimality of rotor-router graph exploration," Information Processing Letters, vol. 127, pp. 17-20, Nov. 2017, doi: 10.1016/j.ipl.2017.06.010.

[24] I. Zelinka and G. Chen, Evolutionary algorithms, swarm dynamics and complex networks, vol. 26. Berlin, Heidelberg: Springer Berlin Heidelberg, 2018.

[25] L. N. Couto, A. R. Backes, and C. A. Z. Barcelos, "Texture characterization via deterministic walks' direction histogram applied to a complex network-based image transformation," Pattern Recognition Letters, vol. 97, pp. 77-83, Oct. 2017, doi: 10.1016/j.patrec.2017.07.013.

\section{BIOGRAPHIES OF AUTHORS}

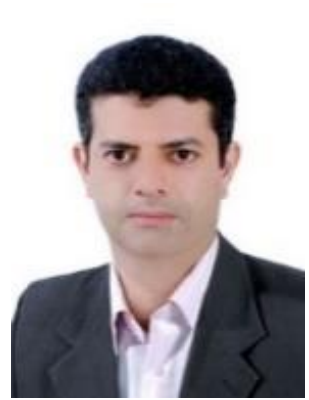

Ali Ebrahimi (D) 8I SC P obtained his B.Sc. in Applied Mathematics from Yazd University in 1996 and his M.Sc. in Computer Software Engineering from Yazd Azad University in 2014. $\mathrm{He}$ is currently a Ph.D. candidate in Computer Software Engineering at Islamic Azad University of Maybod and is working as the head of ICT at Yazd Gas Company. He can be contacted at email: ali.ebrahimi.yazd@gmail.com. 

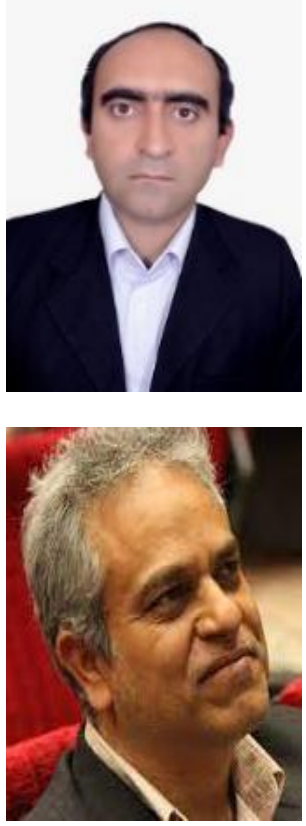

Kamal Mirzaie (iD) SC SC $\mathrm{P}$ obtained his B.Sc. in Computer Engineering from Iran University of Science and Technology (IUST) in 2003 and his M.Sc. in Computer Engineering from University of Isfahan (UI) in 2005. He obtained his Ph.D. In 2011, he is currently working as an Assistant Professor at the Department of Computer Engineering, Maybod Branch, Islamic Azad University, and Maybod, Iran. His-research interests include cognitive science, soft computing, medical data mining, parallel processing, image processing, and pattern recognition. He can be contacted at email: k.mirzaie@ maybodiau.ac.ir.

Ali Mohammad Latif (iD) SC P obtained his first degree in Electronic Engineering from Isfahan University of Technology in 1993 and his M.Sc. in Electronic Engineering from Amirkabir University of Technology in 1996. In 2001, he joined the academic staff at electrical engineering department of Yazd University. He worked as a Ph.D. candidate at the University of Isfahan where he obtained his Ph.D. In 2011, he joined the academic staff at computer department of Yazd University. He can be contacted at email: alatif@yazd.ac.ir. 